Ann. Zootech., I978, 27 (I), 33-45.

\title{
Etude des activités d'un troupeau de poulinières de trait et de leurs poulains au pâturage
}

\author{
W. MARTIN-ROSSET, M. DOREAU et Joëlle CI,OIX \\ Laboratoire de la Production de Viande, \\ Centre de Recherches de Clermont-Ferrand, I.N.R.A. \\ Theix, 63t10 Beaumont (France)
}

\section{Résumé}

Les activités alimentaires au pâturage d'un troupeau de juments rle races lourdes, en partie suitées, ont été observées visuellement du lever du jour à la tombée de la nuit pendant 15 journées.

Les juments effectuent de 3 à 5 cycles de pâturage par jour; le premier commence ${ }_{5}$ à $90 \mathrm{mn}$ avant le lever du soleil; le dernier qui est le plus long s'achève 2 à $5 \mathrm{~h}$ après le coucher du soleil selon le mois de la saison. La durée totale de pâturage a été en moyenne de $592 \pm 91 \mathrm{mn}$, avec de fortes différences entre journées. Faible au cours des premières semaines suivant la mise bas, la durée de pâturage augmente jusqu'à la $3^{e}$ semaine et devient supérieure d'une demi-heure à celle des juments non allaitantes.

Les poulains effectuent un nombre de tétées berucoup plus élevé que celui du vean. Toutefois, la fréquence de tétées diminue rapidement avec l'âge alors que le temps de pâturage augmente.

\section{Introdu :tion}

Le comportement des Équidés aı pâturage, après les premièresobservations de Blechschmid (r933) sur der:x juments de trait, a fait récemment l'objet de divers travaux, centrés sur le comportement social d'Équidés sauvages tels le Zèbre (KLINGEL, I967, I972) ou à demi-sauvages tels le Mustang (FEIST, I97I), le Poney New-Forest (TYLER, 1972) et le Cheval camarguais (GoLdSChmidTRoTHSCHILD, I974; SERENI, I977); sur 1'utilisation du territoire ('TAYLOR, I954; Hafez, Williams, Wifrzbowski, I962; ÖDBERG et Francis-SMith, I977); ou sur les préférences alimentaires (ARCHER, I97I, I973; TYLER, I972; ROGALSkI, I974).

Les activités alimentaires (durées, rythmes) n'ont été observées que sur chevaux de selle par RoGALSKI (I970, I974, I975) et CoUGoUILLE (I976). Le comportement alimentaire du poulain sous la mère a été étudié par FróHLICH-SCHWARZNecker (I921), Bi,EChSChMidt (I933), Neseni et al. (I958), Barmincev (I958), 
Feist (I97 I), Tyler (I972), Rogalski (I973), Schoen, Banks et Curtis (I976); la plupart de ces auteurs n'ont mesuré que la fréquence et la durée des tétées. Les résultats obtenus, souvent contradictoires, traduisent probablement les importantes variations dues à la race et à l'âge du poulain, au mode de conduite de la mère, mais également à la méthode d'observation.

I'objet de notre étude est de décrire, à l'aide d'un enregistrement visuel, selon la méthode utilisée par Perrit (I969), le comportement d'un troupeau de juments, allaitantes ou non, exploitant d'avril à novembre, des pâturages d'altitude, afin de préciser l'emploi du temps journalier des juments et de leurs poulains ainsi que la fréquence de tétée.

\section{Matériel et méthodes}

Nous avons observé au cours de 2 années consécutives (r973-74) les activités d'un troupeau de 19 à 25 juments de races de trait (tabl. I). Les juments étaient âgées en moyenne de 5 à 6 ans, les poulinages ont eu lieu le $2 \mathrm{I} / 4 \pm 2 \mathrm{I} \mathrm{j}$. Le troupeau était conduit en plein air toute l'année. L'hiver, il recevait du foin ad libitum et un apport limité d'aliment concentré. La mise à l'herbe a été effectuée miavril à Theix à $850 \mathrm{~m}$ d'altitude sur de bonnes prairies. Début juin, le troupeau est monté en estive dans la Chaîne des Monts Dore (I $500 \mathrm{~m}$ d'altitude) pour pâturer jusqu'à mi-novembre, des prairies de qualité médiocre exploitées en rotation ( 2 passages par parcelle) et composées de 40 à $50 \mathrm{p}$. cent de graminées ( 3 à $5 \mathrm{p}$. cent de Nard), de 40 à $45 \mathrm{p}$. cent d'espèces herbacées non graminéennes et de $\mathrm{I}_{5}$ à 20 p. cent de ligneux (LOISEAU, I974).

Nous avons défini les activités suivantes :

- pâturage : la tête du cheval est au niveau du sol,

- repos debout : le cheval est en station debout, immobile.

- repos couché : Le cheval est couché et inactif.

- flânerie : terme englobant toutes les autres activités: déplacements, jeux, soins corporels, etc...

- tétée.

Ces conventions facilitent l'observation visuelle; cependant, il faut noter que la durée d'ingestion ne coïncide pas exactement avec la durée de pâturage définie ci-dessus : celle-ci correspond au choix, à la préhension et à la mastication de l'herbe.

Les animaux ont été observés pendant $1_{5}$ journées réparties entre 1973 et I974, du point du jour à la tombée de la nuit; quelques observations nocturnes ponctuelles ont été effectuées. Toutes les tétées des poulains ont été notées; les autres activités ont été enregistrées individuellement à intervalles de $5 \mathrm{mn}$ pour les juments allaitantes et leurs poulains, globalement à intervalles de $30 \mathrm{mn}$ pour les juments non allaitantes.

En supposant constante l'activité du troupeau entre deux observations successives, notus avons calculé :

- le temps journalier consacré par chaque animal à une activité $a$ :

$$
\mathrm{T} a=\mathrm{N} a \times d
$$

$\mathrm{N} a$ : nombre de fois où l'animal a été observé effectuant l'activité $a$; $d$ : intervalle entre deux observations successives. 


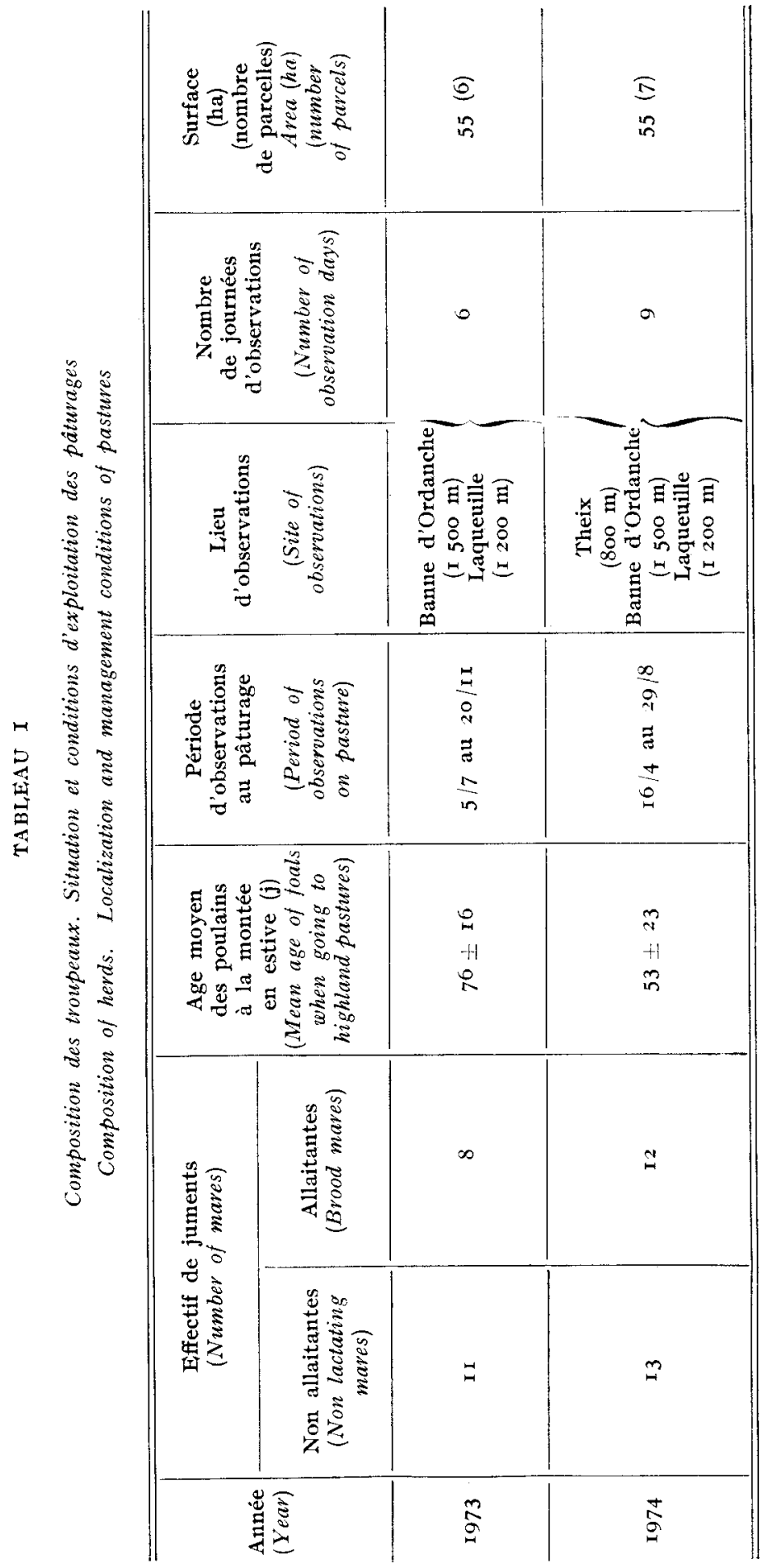


- la part relative ou fréquence de chaque activité du troupeau, définic par le rapport :

$$
\frac{\text { nombre d'animaux exerçant cette activité }}{\text { nombre d'animaux présents }}
$$

Pour chaque journée d'observations, nous avons tracé les histogrammes de fréquence du pâturage et de la tétée. Le pâturage s'étant révélé cyclique, nous avons distingué les différents cycles en supposant, comme Perrir (I972), que deux périodes voisines de pâturage présentant des effectifs $E_{1}$ et $F_{2}$ sont deux cycles distincts si $30 \mathrm{mn}$ au moins les séparent, pendant lesquelles le nombre d'animaux qui pâturent est inférieur à $\frac{\mathrm{E}_{1}+\mathrm{E}_{2}}{4}$.

\section{Résultats}

Activité des juments (fig. I, 2)

\section{Cycles d'activité}

Les juments commencent à pâturer de $\mathrm{I}_{5}$ à $90 \mathrm{mn}$ avant le lever du soleil, en particulier au printemps et en été. Elles effectuent de 3 à 5 cycles de pâturage

\begin{tabular}{|c|c|c|c|}
\hline Dotes & & 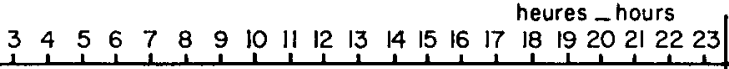 & $(\mathrm{mn})$ \\
\hline $18 / 4$ & $\begin{array}{l}\text { J.N.A. } \\
\text { J.A. }\end{array}$ & 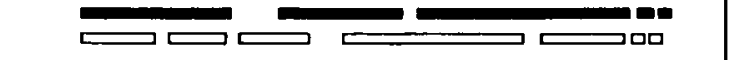 & $\begin{array}{l}600 \\
585\end{array}$ \\
\hline $15 / 5$ & $\begin{array}{l}\text { J.N.A. } \\
\text { J.A. }\end{array}$ & $\rightleftarrows$ & $\begin{array}{l}639 \\
645\end{array}$ \\
\hline $11 / 6$ & $\begin{array}{l}\text { J.N.A. } \\
\text { J.A. }\end{array}$ & ש & $\begin{array}{l}638 \\
693\end{array}$ \\
\hline $19 / 6$ & $\begin{array}{l}\text { J.N.A. } \\
\text { J.A. }\end{array}$ & ه & $\begin{array}{l}643 \\
624\end{array}$ \\
\hline $9 / 8$ & $\begin{array}{c}\text { J.N.A } \\
\text { J.A }\end{array}$ & ש & $\begin{array}{l}555 \\
554\end{array}$ \\
\hline $29 / 8$ & $\begin{array}{l}\text { J.N.A } \\
\text { J.A }\end{array}$ & 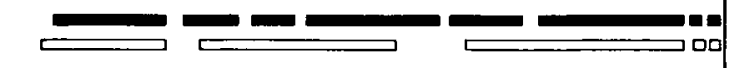 & $\begin{array}{l}628 \\
610\end{array}$ \\
\hline $4 / 9$ & $\begin{array}{l}\text { JN.A. } \\
\text { J.A. }\end{array}$ & $\rightleftarrows$ ॠ & $\begin{array}{l}552 \\
555\end{array}$ \\
\hline $13 / 9$ & $\begin{array}{l}\text { J.N.A. } \\
\text { J.A. }\end{array}$ & ם & $\begin{array}{l}583 \\
622\end{array}$ \\
\hline $31 / 10$ & $\begin{array}{l}\text { JNA } \\
\text { J.A }\end{array}$ & $\rightleftarrows$ & $\begin{array}{l}518 \\
550\end{array}$ \\
\hline $20 / 11$ & $\begin{array}{l}\text { J.N.A } \\
\text { J.A. }\end{array}$ & ב & $\begin{array}{l}444 \\
464\end{array}$ \\
\hline
\end{tabular}
diurne (fig. I). Le cycle matinal est court $(2$ à $3 \mathrm{~h}$ ) et s'achève tôt, entre $6 \mathrm{~h} 30$ et

HIG. I. -... Horaires de paturage du troupeau de juments (Grasing patterns of a mare's herd).

J.N.A. : juments non allatantes (non lactating mares).

J.A. : juments allaitantes (brood mares).

N.B. : Ces 10 journées n'ont pas présenté de perturbations climatiques (pluies on jortcs chaleurs). These 10 days were chosen for their good climatic conditions (nother rain nor havd heat). 
$9 \mathrm{~h}$, surtout lorsque les jours sont longs et chauds, en juin-juillet-août. Ensuite, au cours de la journée, le troupeau pâture pendant 4 à $6 \mathrm{~h}$, réparties en I à 3 cycles, selon le mois considéré, quel que soit l'état physiologique de la jument. Le nombre et la durée des périodes de repos varient respectivement de 2 à 4 et de 30 à I 20 mn. C'est en début d'après-midi, entre $\mathrm{I}_{3} \mathrm{~h}$ et $\mathrm{I} 7 \mathrm{~h}$, qu'a lieu la période principale de repos. Le cycle de pâturage de la soirée lui succède. Il est systématiquement le plus long. Il commence d'autant plus tôt que la période diurne diminue et il s'achève tard, entre $2 \mathrm{I} \mathrm{h}$ et $23 \mathrm{~h}$, soit de 2 à $5 \mathrm{~h}$ après le coucher du soleil; il peut durer de 5 à $7 \mathrm{~h}$. Ces résultats concordent assez bien avec les observations de TyLER (I972), au moins en ce qui concerne les cycles de pâturage effectués par les Poneys New Forest au début et à la fin de la journée.

Nous n'avons pu observer l'activité nocturne des juments que de manière très ponctuelle, mais suffisante pour constater qu'elles pâturent la nuit. Chez le Poney Pottock, Ruckebusch, Vigroux, Candau (I976) estiment que la durée de l'ingestion nocturne d'herbe distribuée à l'auge correspond à $28,8 \mathrm{p}$. cent de $1 \mathrm{a}$ période nocturne. Par ailleurs, selon KL,INGEI, (I967), le zèbre effectue 2 à 3 cycles de pâturage nocturne au cours des nuits de clair de lune.

\section{Durée des activités}

Le temps de pâturage diurne est élevé tout au long de la saison : $592 \pm 9 \mathrm{I} \mathrm{mn}$ pour une durée moyenne d'observations de $800 \mathrm{mn}$, soit $67 \mathrm{p}$. cent du temps diurne (de 62 à $77 \mathrm{p}$. cent). Ces résultats sont tout à fait comparables aux données obtenues pat Ruckebusch, Vigroux et Candau (1976) chez les Poneys Pottock recevant de l'herbe coupée à l'auge à volonté. En revanche, ils sont inférieurs de 5 à I 5 points à ceux enregistrés au pâturage par RoGALSKI (I970) qui ne disposait que de surfaces restreintes, (2-3 ha), et rentrait les animaux au box le soir. Le faible temps de pâturage observé par CougourLLE (I976), $56 \mathrm{p}$. cent sur une prairie temporaire de bonne qualité est attribué au temps réduit consacré au tri.

Le temps de repos total a été de $2 \mathrm{I} 6 \pm 50 \mathrm{mn}$ environ, soit seulement $22 \mathrm{p}$. cent du temps diume. La jument se couche peu, $28 \pm \mathrm{I} 8 \mathrm{mn}$, soit 3 p. cent de la phase diurne (fig. 2). En fin, les flâneries représentent $66 \pm 28 \mathrm{mn}$, soit 8 p. cent du temps diurne.

\section{Facteurs de variations}

Les variations du temps diurne sont davantage liées aux différences entre journées qu'aux différences individuelles : le carré moyen inter-journées est 25 fois plus élevé que le carré moyen intrajournées. Cette homogénéité relative du temps de pâturage des individus composant le troupeau est d'autant plus surprenante qu'à l'écurie DOREAU (en préparation) a noté l'importance des variations individuelles du niveau d'ingestion. L'organisation des activités alimentaires du cheval à l'herbe en cycles peut sans doute expliquer cette différence.

Les fluctuations des activités entre journées sont liées à la saison, aux condi tions météorologiques, à l'état physiologique de la poulinière, sans qu'il soit facile de dissocier l'influence de ces différents facteurs.

Lorsque le temps est chaud (ex. journée du 9-8-73), les juments réduisent leur pâturage diurne de 30 à $4^{\circ} \mathrm{mn}$ environ. Quand le temps devient orageux et que les juments disposent d'une zone d'ombrage, le pâturage diurne peut, à l'image de ce 


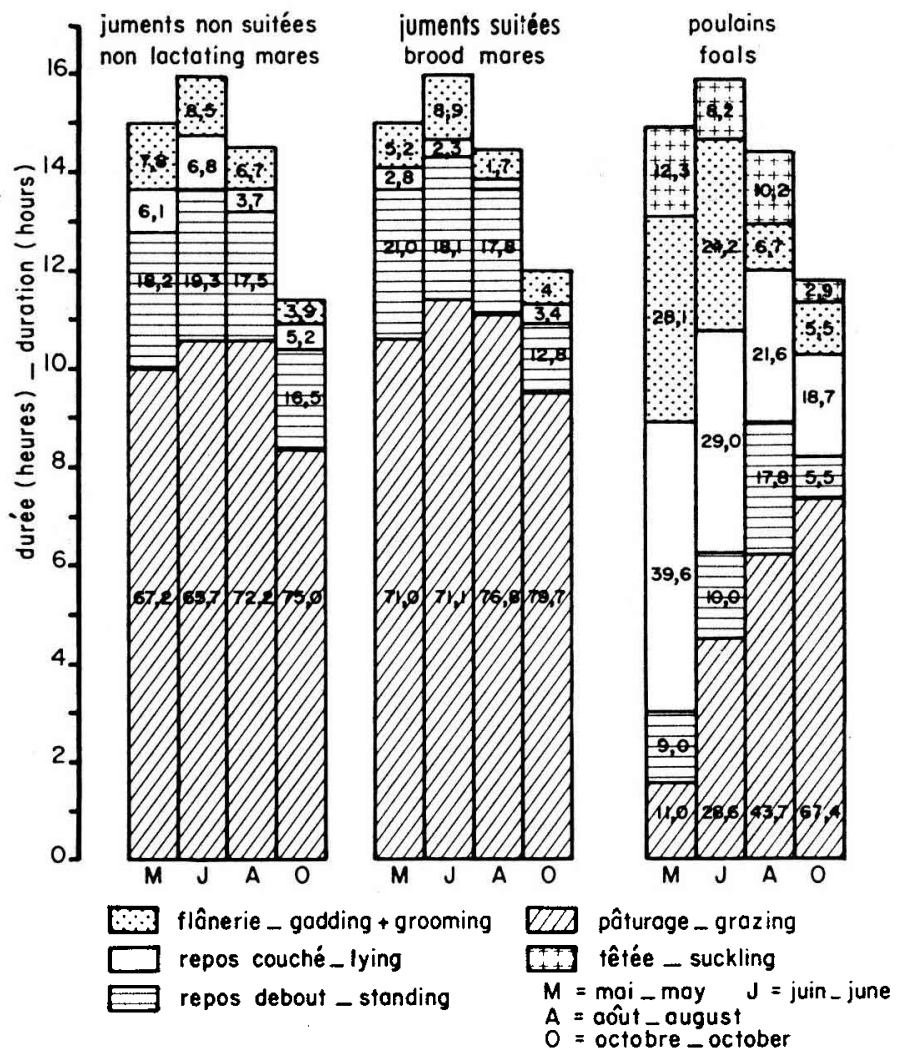

FIG. 2. - Évolution avec la saison des temps d'activité des juments suitées et de leurs poulains, et des juments non suitées.

Seasonal variations of diurnal activity times in brood mares and their foals, and in no lactating mares.

N.B. : Les durées totales représentent le temps diume d'observation. Les nombres indiquent la répartition de ce temps diurne entre les différentes activités.

Total durations represent the diurnal observation time. Numbers show the distribution of this diurnal time between different activities.

que TyLER (I972) a observé chez le Poney New Forest, ne représenter que 32 p. cent de la phase diurne, soit $6 \mathrm{~h}$ au lieu de II h (exemple journée du 28-6-73). Les juments se reposent alors essentiellement debout. Le pâturage ne reprend qu'après le coucher du soleil et doit se poursuivre pendant une grande partie de la nuit.

Les juments semblent réagir différemment à la pluie selon 1'intensité des précipitations. Quand la pluie est faible ou intermittente, le temps de pâturage diurne s'accroît au détriment du temps de repos de près d'une heure, soit II à I2 $p$. cent de la période diurne; les cycles de pâturage sont moins marqués et les horaires peuvent être quelque peu décalés, le tout donnant l'impression d'un pâturage continu. En revanche, lorsque les précipitations deviennent plus fortes, les juments ont tendance à réduire le temps de pâturage et à le différer au lendemain.

Les juments sont également sensibles à la diminution de la qualité et dela quantité de l'herbe au cours de la saison. Au début de la saison de pâturage, l'herbe 
étant abondante, elles effectuent de 4 à 5 cycles de pâturage bien distincts (fig. I). Les périodes de repos et de flâneries sont nombreuses. Leur durée augmente jusqu'à fin août, puis diminue en automne car les cycles de pâturage du milieu de la journée fusionnent rapidement. Le temps de pâturage, exprimé par rapport à la durée d'observation, augmente alors régulièrement et se prolonge tard après le coucher du soleil : 2 à $3 \mathrm{~h}$ (fig. I, 2); ce fait a également été signalé par RoGALSKI (1975).

Le temps de pâturage des juments allaitantes est seulement de $475 \pm 68 \mathrm{mn}$ au cours de la première semaine qui suit la mise-bas, soit respectivement |roo à I $70 \mathrm{mn}$ et 75 à $125 \mathrm{mn}$ de moins que celui enregistré chez les juments allaitant depuis I mois et les juments non suitées exploitant simultanément les mêmes parcelles. Cela est sans doute lié au nombre élevé de tétées du poulain au cours des premières semaines.

Un mois après le poulinage, le temps de fâturage de la poulinière est plus. long, en moyenne de $30 \mathrm{mn}$, que celui des juments non suitées exploitant la même herbe au même stade. Cette différence se maintient pratiquement jusqu'au sevrage. Les liaisons avec l'âge, le poids et le nombre de tétées des poulains sont faibles; les coefficients de corrélation varient de 0,1 à 0,2 .

Par ailleurs, le temps de pâturage des juments semble indépendant de leur poids vif.

\section{Activités des poulains}

Tétées (fig. $3^{a}$ )

L'activité du poulain en liberté avec sa mère au pâturage est dominée par la tétée. Le nombre de tétées observé pendant la phase diurne est élevée, en moyenne 20 tétées par jour, de la naissance au sevrage. Il est faible et irrégulier, au cours des 24 à $48 \mathrm{~h}$ qui suivent la naissance, car il dépend beaucoup de la rapidité de l'apprentissage du poulain à la tétée et du degré d'instinct maternel de la poulinière.
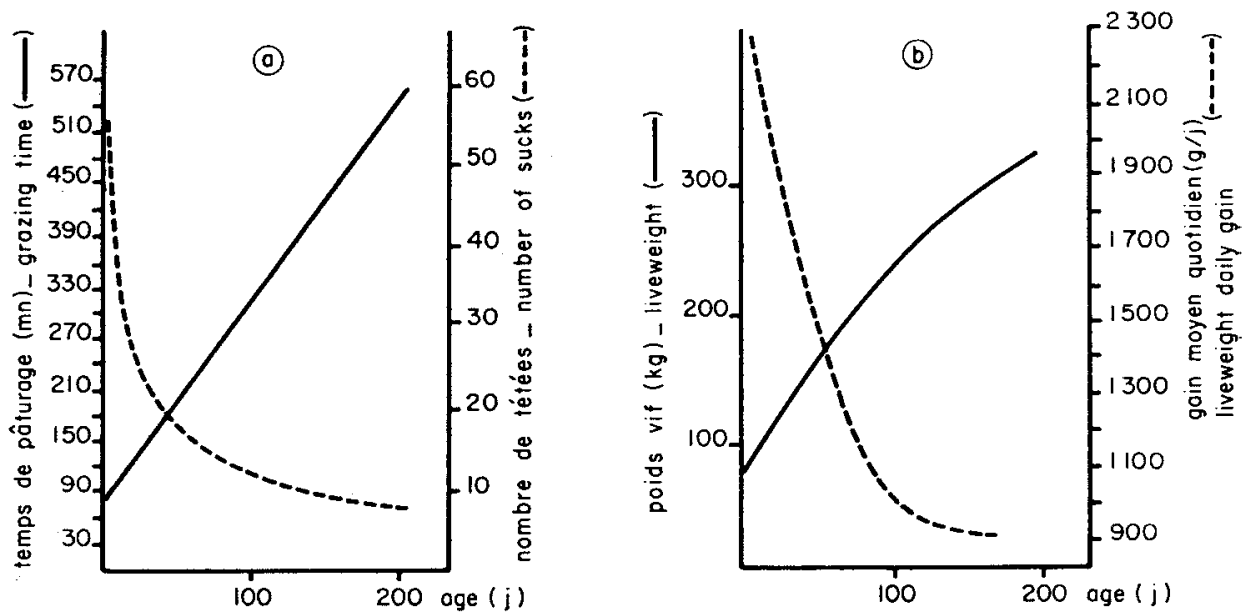

FIG. 3. - Évolution du nombre de tétées, du temps de pâturage, de la vitesse de croissance et du poids vit du poulain de trait en fonction de l'age.

Variation with age in the number of suckings, grazing time, growth vate and liveweight of heavy foals. 
Il atteint un maximum de 3 à 4 tétées par heure dès la première semaine, puis diminue jusqu'au sevrage selon une courbe de type exponentiel (fig. $3 a$ ). L'équation de la courbe ajustée en coordonnées semi-logarithmiques est de la forme :

$$
y=-0,2 \mathrm{I} \log x+56,37 \quad r=\mathrm{Co}_{4}^{* * *} \quad n=\mathrm{I} 5 \mathrm{o.}
$$

où $y=$ nombre de tétées par jour.

$x==$ âge $(j)$.

Ces résultats concordent assez bien avec les observations enregistrées sur le Cheval par FRöHLICH-SCHWARZNECKER (I92 I), BLECHSCHMIDT (I 933), BARMINCEV (I958), ou plus récemment par FEIST (I97I) sur le Mustang. La fréquence de tétée que nous avons enregistrée est cependant légèrement inférieure à celle observée par Tylen (1972) et surtout n'est pas maximum au cours des $48 \mathrm{~h}$ suivant la mise bas. Par ailleurs, il convient de signaler que l'évolution du nombre de tétées que nous avons observée est en contradiction avec les données enregistrées chez le poulain de selle par NESENi et al. (I958). Ces auteurs rapportent en effet que le nombre de tétées chez le potrlain de selle augmenterait de la naissance jusqu'au pic de lactation qu'ils situent vers $2-3$ mois, puis diminuerait au-delà. Il est difficile toutefois d'expliquer cette différence car nous n'avons pas observé les tétées nocturnes, et parce que la méthodologie utilisée pour effectuer ces mesures n'est pas indiquée clairement par NESENI et al.

Les tétées ne semblent pas se situer à des moments précis de la phase diurne, contrairement aux observations de Schoen, BANks et CuRTIS (I976), ni être liées à une activité particulière de la mère, sauf au cours des $I_{5} \mathrm{j}$ suivant la mise bas. Pendant cette période, la jument prolonge le temps de repos debout au détriment du pâturage pour faciliter la tétée du jeune. Les tétées peuvent être déclenchées aussi bien par le poulain que par la poulinière. Au cours des premières semaines suivant la mise bas, elles sont systématiquement précédées d'une période de repos couché chez le poulain, et leur signal est souvent donné par la mère. Le processus semble s'inverser quand l'âge du poulain augmente.

La jument reste en principe immobile lorsque le poulain sollicite la tétée. Dans le cas contraire, pâturage ou déplacement, le poulain effectue un ou plusieurs passages au poitrail pour immobiliser la mère. Au cours des premières semaines suivant la mise bas, la tétée est interrompue le plus souvent par le poulain, sauf cas particuliers... Lorsque le poulain prend de l'âge, la mère met fin à la tétée en se déplaçant ou en mordant, surtout lorsqu'on approche du sevrage.

La durée de la tétée est courte en moyenne (40 à go sec.), mais son évolution n'a pu être suivie de façon précise. Les données disponibles à ce sujet sont contradictoires. La durée de la tétée resterait, de la naissance au sevrage, constante chez le Mustang (FEIST, I97I) et diminuerait chez les Chevaux de selle (NESENI et al., I958) et chez le Poney ('TYLER, I 972). Ces derniers résultats semblent plus logiques, puisque Rogalski (1973) note que la capacité de déglutitionl du poulain augmente avec l'âge.

\section{Pâturagc. Repos et flâneries}

Au cours des premières semaines suivant la naissance, le poulain partage ses activités entre les jeux et le repos, surtout couché; le repos debout étant peu important. Le repos couché et les flâneries représentent respectivement 40 et $28 \mathrm{p}$. cent de l'emploi du temps diurne du poulain à l'âge de I mois (fig. 2). 
Cépendant, dès le plus jeune âge ( 5 à Io $\mathrm{j}$ ), le poulain s'intéresse à l'herbe. Il s'agit plus d'un " mâchouillage " que d'un pâturage effectif, ainsi que l'ont observé HaFEz, Wilimams et Wierzbowski (I962) et Rogaiski (I973). I)e plus, jusqu'à l'âge de I mois environ, il ingère fréquemment les crottins maternels. Puis cette coprophagie régresse au fur et à mesure que l'ingestion d'herbe augmente et devient négligeable à un mois, lorsque le développement de la flore microbienne est achevé (BAINTNER, OCsAG, Fülor, I97I). A l'âge d'un mois, le poulain consacre i I p. cent de la phase diurne à pâturer (fig. 2), soit un temps égal à $15 \mathrm{p}$. cent environ de celui de sa mère. Le pâturage des poulains devient alors cyclique comme celui des mères, mais les cycles sont peu nombreux et ils débutent après celui des poulinières et s'achèvent avant. Dès l'âge de 2 à 3 mois, le nombre de cycles de pâturage est sensiblement égal à celui de la mère et les horaires sont analogues.

Le temps de pâturage journalier ( $y$ en $\mathrm{mn}$ ) mesuré de la naissance au sevrage vers $190 \mathrm{j}$ augmente de manière linéaire avec :

- 1'âge $(x$ en j) (fig. $3 a)$ :

$$
y=2,28 x+09,22 \quad r=0,82 * * * \quad n=\mathbf{1} 50 \text {, }
$$

- et le poids du poulain ( $x$ en $\mathrm{kg}$ )

$$
y=1,79 x-100,99 \quad r=0,85^{* * *} n=\mathbf{1} 50 .
$$

en même temps que le nombre de tétées $(x)$ diminue :

$$
y=-9.54 x \div 428,99 \quad r=-0,69^{* * *} \quad u=\mathrm{r}_{5} \text { \%, }
$$

mais il est indépendant du temps de pâturage des mères.

A partir du mois d'août, alors que les poulains sont âgés de 4 mois environ, le temps de pâturage représente $44 \mathrm{p}$. cent de la période diurne, soit $57 \mathrm{p}$. cent de celui de la mère. Au sevrage, il atteint $80 \mathrm{p}$. cent de celui de la poulinière. L'augmentation du temps de pâturage s'effectue aux dépens des temps de repos debout, couché et de flâneries; ceux-ci ne représentant plus respectivement que 22 et 7 p. cent de la phase diurne. On commence alors à observer un pâturage nocturne.

\section{Méthodologie d'obseriations}

Nous avons déterminé, pour une journée d'observations, la taille optimale de l'échantillon de juments allaitant depuis plus de ${ }_{5} \mathrm{j}$ qui permet d'estimer le temps de pâturage diurne (à 30,45 et $60 \mathrm{mn}$ près) avec un seuil de probabilité de 95 p. cent. Le calcul a été effectué pour quatre fréquences d'observations : 5, Io, I5 et $30 \mathrm{~mm}$. L'intervalle de confiance, autour d'une moyenne $\mathrm{X}$, est définie par: $\mathrm{I} \pm \frac{t \sigma}{\sqrt{n}}$. Ilans notre cas :

$n=\frac{t_{0.05}^{2} \sigma^{2}}{d^{2}}$

$d=$ Précision recherchée $(30,45$ ou $60 \mathrm{mn})$.

$t_{0,05}=$ Valeur tabulée du $t$ de Student.

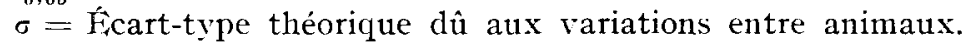


Nous avons choisi comme estimateur de $\sigma^{2}$ la variance intra-jours $\Gamma$ :

$$
\Gamma=\frac{\Sigma_{i} \Sigma_{j}\left(\mathrm{X}_{i j}-\mu_{j}\right)^{2}}{\nu}
$$

$\mathbf{X}_{i j}=$ Temps de pâturage diurne de l'animal $j$ le jour $i$ pour la fréquence d'observations considérée.

$\mu_{j}=$ Moyenne journalière théorique du temps de pâturage diurne.

$\nu=$ Nombre de degrés de liberté.

Lorsque la fréquence d'observations est de $5 \mathrm{mn}, \ell_{j}$ est estimé par $m_{j}=\frac{\Sigma \mathrm{X} i_{j}}{n_{j}}$. Le nombre de degrés de liberté est alors :

$\nu=\left(\sum_{i} n_{j}\right)-p$.

$n_{j}=$ nombre d'observations effectuées le jour $i$.

$p=$ nombre de jours d'observations.

Nous obtenons ainsi deux relations :

- Fréquence d'observations de $5 \mathrm{mn}$ :

$$
n=\frac{t^{2}{ }_{0,05}}{d} \cdot \frac{\sum_{i} \sum_{j}\left(\mathrm{X}_{i j}-m_{j}\right)^{2}}{\left(\sum_{j} n_{j}\right)-p}
$$

-.- Autres fréquences d'observations

$$
n=\frac{t^{2}{ }_{0,05}}{d} \cdot \frac{\sum_{i} \sum_{j}\left(\mathrm{X}_{i j}-m_{j}\right)^{2}}{\sum_{j} n_{j}}
$$

Ceci nous a permis d'établir le tableau suivant, après avoir arrondi les valeurs de $n$ à l'entier supérieur (tabl. 2).

\section{TABLEAU 2}

Nombre de juments allaitantes à observer a fin d'estimer la durée de pâturage avec une précision donnée Number of brood mares necessary to estimate time spent grazing with a giving precision

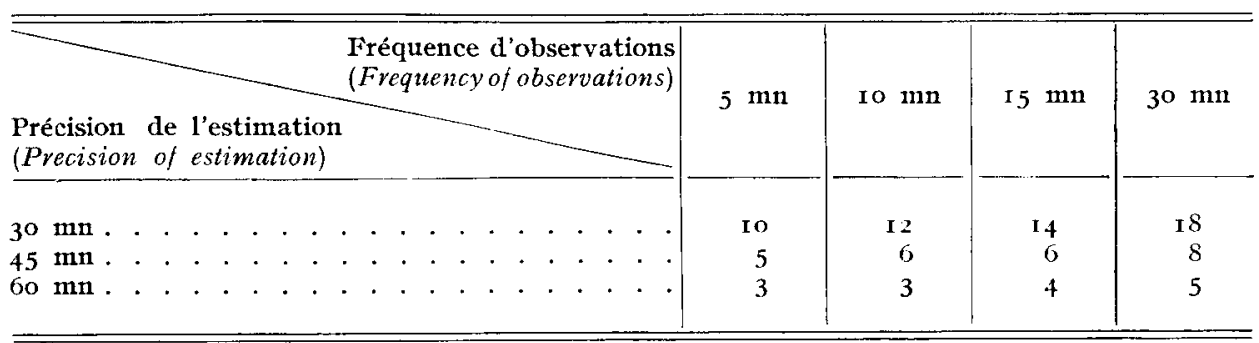

Il est donc préférable d'observer un nombre réduit d'animaux au sein du troupeau et de multiplier les journées d'enregistrement. 


\section{Discussion}

L'activité des troupeaux de juments allaitantes et de leurs poulains, exploitant des pâturages d'altitude, est cyclique comme celle des troupeaux de vaches allaitantes, conduits sur des pâturages analogues (PETIT, I972). L'emploi du temps est cependant différent et leur activité n'est pas uniquement diurne. En effet, en dehors du cycle matinal de pâturage qui s'effectue à des horaires similaires chez les chevaux et les bovins, les juments pâturent à partir de 8-9 h et jusqu'à $\mathbf{I}_{4}-\mathrm{I} 5 \mathrm{~h}$, au cours de $I$ à 4 cycles. Le dernier cycle se prolonge bien après le coucher du soleil. Le pâturage des juments représente $67 \mathrm{p}$. cent du temps diurne contre $5^{\mathrm{I}} \mathrm{p}$. cent chez la vache allaitante. RoGALSKI (I974) a observé que les temps de pâturage des chevaux de selle, exploitant simultanément avec des moutons et des bovins des parcelles comparables, étaient sensiblement équivalents au temps total de pâturage et de rumination des moutons et des bovins. Ces résultats sont d'ailleurs confirmés par les enregistrements effectués pendant $24 \mathrm{~h}$ à l'auge par DOREAU (en préparation) sur des chevaux de trait recevant du foin de prairie naturelle à volonté et de l'avoine $(2 \mathrm{~kg} / \mathrm{j})$ et par DULPHY (I 972$)$ sur des moutons recevant à volonté un foin de graminées : la durée d'ingestion des chevaux n'est que légèrement inférieure à la durée de mastication (ingestion + rumination) observée sur des moutons.

Le temps de pâturage diurne des juments allaitantes paraît également lié, comme chez les vaches allaitantes, d'une part à la quantité et à la qualité de l'herbe disponible, d'autre part, à la longueur du jour. Il augmente avec l'avancement en saison, lorsque la quantité et sans doute la qualité de l'herbe diminuent sansqu'il soit facile de séparer l'influence respective de ces deux facteurs de variation. I1 diminue par ailleurs, avec le raccourcissement de la phase diurne malgré un allongement des cycles de pâturage. Mais, rapportée à la longueur du jour, la proportion de temps consacrée au pâturage augmente. La différence entre les temps de pâturage des juments allaitantes et non allaitantes traduit une bonne faculté d'adaptation aux besoins.

La fréquence des tétées chez le poulain, de la naissance au sevrage, est exceptionnellement élevée et bien supérieure à celle observée chez le veau. La faible capacité de la mamelle de la jument (environ 21 dans le cas de la jument de trait, d'après Chashoin et Mironenko, I955; Storch, I969) nécessite en effet une vidange fréquente et rapide. Cette particularité rend très difficile toutes mesures d'estimation de la production laitière par pesées du jeune, avant et après tétée, comme chez le veau. C'est pourquoi toutes les estimations de la production laitière des juments qui ont pu être faites, à l'aide de cette méthode, sont probablement sujettes à caution.

Il convient néanmoins de remarquer que le poulain semble capable, d'une part d'augmenter son temps de pâturage lorsque la production laitière de la mère diminue et, d'autre part, d'ajuster son temps de pâturage à ses besoins lorsque la quantité et la qualité de l'herbe diminuent (fig. $3 a-b$ ). C'est sans doute pourquoi le poulain peut réaliser, tout au long de la saison, un croît de la naissance au sevrage de I $300 \mathrm{~g} / \mathrm{j}$ (MARTIN-Rossér, résultats non publiés) bien supérieur à celui des veaux (750 à $900 \mathrm{~g}$; PETIT, 1974) conduits pourtant sur de meilleurs pâturages à une altitude moins élevée. 


\section{Summary \\ Grazing behaviour of a herd of heary brood mares and their foals}

The grazing behaviour of a herd of 8 heavy brood mares and their foals and 12 non lactating mares was studied. Recordings were made during 15 days between $A$ pril and November at intervals of $5 \mathrm{mn}$ from sunrise to sunset. Only diurnal activities were measured (Table I).

The herd was grazing during 3 to 5 cycles (fig. I). Brood mares were grazing during 592 $\pm 9 \mathrm{rmn}$. The distribution of the activities of the mares: grazing, standing, lying and gadding + grooming (other activities) was $67,22,3$ and 8 p. cent, respectively. The inter-day variations were much higher than the intra-day variations. The differences between days were essentially related to season and climatic conditions: grazing time decreased from the end of August; it seemed to be reduced by strong heat or violent rain (fig. 2).

The differences between animals can be partially explained by the physiological state and, in the brood mares, by the variation in their requirements during lactation. The grazing time of mares after foaling was short. It increased until the $3^{\text {rd }}$ week and then exceeded by $30 \mathrm{mn}$ that of the non lactating mares. This difference persisted until weaning.

The number of diurnal sucks in the foal was very high 48 hours after birth, but decreased rapidly with age $(y=0,21 \log x+56,37)$, whereas grazing time increased $(y=2,28 x+69,22)$ (fig. $3 a$ ). These observations are related with the fact that in the foal the growth rate decreases with age (fig. $3 b$ ).

On the basis of the experimental results, it was possible to determine the minimum number of animals necessary for estimating the grazing time of a given day and with a given accuracy (Table 2).

The present results have been compared with those obtained in cattle and emphasize some particularities in horses: a long grazing time in the mare, a high frequency of sucks and a high growth rate in the foal.

\section{Références bibliographiques}

ARCHER M., I97I. Preliminary studies on the palatability of grasses, legumes and herbs to horses. Vet. Rec., 89, $23^{6-240 .}$

ARCher M., 1973. The species preferences of grazing horses. J. Br. Graslld Soc, 28, i23-128.

Baintner K., OCsag I., Fülop S., I97I. Produits de fermentations dans les fèces de poulains. (en Hongrois). Allatenyésztés, 20, 83-86.

Barmincev J. N., 1958. Breeding horse for milk under conditions in Kazakhstan. Trudy l. Konf. Mol. Konevod. Kumysod (Mosk), 20-25. (In Anim. Breed. Abstr. 1961, 29, 1905).

Bl.ECHSCHMid H., r933. Experimentaluntersuchungen und Eimittlungen über die Ernahrung des Pferdes auf der Weide. I.andw. Jb., 77, 463-560.

Chashin I., Mroonfenko M., i955. Milk production by mares in Kirgisia Konevodstvo, 25 (6), 19-23 (in Dairy Sci. Abstr., I956, 18, 632).

CovgouiluE B., 1976. Comportement social et utilisation du pâturage par les chevaux. Mom. fin. d'ét., E.N.S.A. Rennes, 28 p. + tab. Station Expérimentale des Haras. 19230 Pompadour.

DOREAU M., 1978. Comportement alimentaire du cheval à l'écurie. Ann. Zootech. (en préparation).

Dulphy J. P., 1972. Iitude de quelques relations entre le mode de conservation da fourrage ingéré et le comportement alimentaire et mérycique des moutons. Ann. Zootech, 21, 429-44r.

FEIST J. D., r97I. Behavior of feral loorses in the Pryor Mountain wild horse range. Master's Theses Univ. Michigan, rzo p.

F́röylich-SchwarzNecker, 1921. Cité par Neseni et al. (1958).

Gol,DSCHMIT-RoTHSCHIL B. von., 1974. Soziale Organisation und Verhalten eines Camargue Pferde Bestandes. Lizenz Arbeit. Univ. Berne, 57 p.

Halez E. S. E., WIILIAMS M., WIERzbowski S., I962. The behaviour of horses in HAFEZ E.S.E. The behaviour of domestic animals, chap. $13,370-396$. Baillière-Tindall-Cox F.d., L.ondon. 
KI.INGFI. H., 1967. Soziale Organisation und Verhalten freilebender Steppenzebras. Z. Tiorpsychol, 24, 580-624.

KLINGEI, H., 1972. Das Verhalten der Pferde (Equidae). In Handbuch der Zoologie, VIII, I $0^{\circ}$ part. 1-68. Walter de Gruyter, Ed. Berlin, New York.

LOISEAU P., I974. (Communication personnelle).

Martin-Rosset W., non publié.

Neseni R. von, Flade, E., Heidilir G., Strgfir H., i958. Milchleistung und Milchzusammensetzung von Stuten im Verlaufe der Laktation. Arch. Tierzucht, 1, 9I-I29.

ÖDnERG F. O., Francis-SMITU K., I 977 . Studies on the formation of ungrazed eliminative areas in fields used by horses. Appl. Anim. Ethol., 3, 27-34.

PETrT M., 1969. Méthode d'observation de l'emploi du temps des troupeaux de vaches allaitantes au pâturage. Ann. Zootech., 18, 221-226.

Perrt M., 1972. Emploi du temps des troupeaux de vaches mères et de leurs veaux sur les pâturages d'altitude de l'Aubrac. Ann. Zootech., 21, 5-27.

PrTIT M., 1974. Vêlage à deux ans dans les troupeaux de vaches allaitantes. In L'exploitation des troupeaux de vaches allaitantes, $23 \mathrm{I}-248$. I.N.R.A.-S.E.I. Versailles, I.T.E.B. Paris.

Rogalski M., 197o. Comportement du Cheval au pâturage (en Polonais). Kon Polski, 5 (4), 26-27.

Rogalskr M., 1973. Comportement du Poulain au pâturage (en Polonais). Proegl. Hodowlany, 41 (5), I4-I5.

ROGALSKI M., I974. The comparison of some characteristic interdependent factors between the sward and the grazing animals. Proc. XII. Intern. Grassld. Congr., Moscou, Sect. pap. "Grassland utilization ", part. II, 582-584.

ROGALSKI M., I975. Effets des conditions climatiques sur le comportement des Chevaux au pâturage (en Polonais). Roczn. Nauk Rolnicz. B. 97 (I). 7-r6.

RuCKEBUSCh Y., Vigroux P., CANDAU Mr., I976. Analyse du comportement alimentaire chez les Équidés. C.R. Journée d'Êtude CEREOPA, 69-72, 16, rue Claude-Bernard, 75005 Paris.

Schoen A. M. S., Banks E. M., Curtis S. E., I976. Behavior of young Shetland and Welsh Ponies (Equus caballus). Biol. Behav. 1, I99-216.

SHRENI J. L., I977. Recherches sur l'évolution de la structure sociale et du comportement alimentaire d'un groupe de chevaux camarguais. Thèse Doct. Spécialité Univ. Aix-Marseille II, $85 \mathrm{p}$.

S'ToRCi R., I969. Mechanical milking of mares. Milchwissenschaft, 24 (3), I45-146.

TAYıoR E. L., I954. Grazing behaviour and helminthic disease. Br. J. Anim. Behav., 2, $6 \mathrm{I}-62$.

TYLER S. J., 1972. The behaviour and social organization of the New-Forest Ponies. Anim. Behav. Monogr., 5 (2), 87-196. 